\title{
Fibroblast Growth Factor 23 (FGF23) is a useful biomarker in the investigation of incidental hypophosphataemia
}

Paul Connelly, lona Galloway, Stephen Gallacher, Andrew Gallagher

Department of Endocrinology \& Diabetes, Queen Elizabeth University Hospital, Glasgow, UK

\section{Case report}

A 77 year old female was referred to endocrinology with an incidental finding of hypophosphataemia $(0.26 \mathrm{mmol} / \mathrm{L})$ on routine bloods. She described a slight unsteadiness on her feet, but denied bone pain or overt muscle weakness. Past medical history included Type 2 Diabetes Mellitus, a left humeral fragility fracture and the subsequent diagnosis of osteoporosis 2 years previously.

At presentation the corrected calcium was slightly elevated (2.64 $\mathrm{mmol} / \mathrm{L})$, which normalised when repeated, with suppression of parathyroid hormone (PTH; $0.8 \mathrm{pmol} / \mathrm{L})$ and adequate 25-hydroxyvitamin $D$ concentrations $(71 \mathrm{nmol} / \mathrm{L})$. Renal function was normal and no paraproteins were detected. Phosphate levels were suboptimal for approximately 3 years, however, had been normal prior to this. FGF23 was found to be significantly elevated (186 RU/ $\mathrm{ml}$; normal range <100).

\section{FGF23 \& phosphate homeostasis}

Phosphate homeostasis is mediated by FGF23, PTH, and $1,25(\mathrm{OH})_{2}$ vitamin D. FGF23 is secreted by osteocytes and acts upon the kidney to':

- Decrease tubular reabsorption of phosphate

- Reduce 1a-hydroxylase expression thereby limiting the activation of vitamin $D$ and intestinal phosphate absorption

Consequently inappropriately high levels of FGF23 result in chronic hypophosphataemia and inadequate bone mineralisation. FGF23 can be measured clinically to aid in the assessment of disorders of phosphate homeostasis and if elevated can be suggestive of FGF23 secreting occult tumours.

\section{Tumour-induced osteomalacia}

Tumour-induced osteomalacia is a rare paraneoplastic disorder characterised by hypophosphataemia due to decreased renal tubular reabsorption of phosphate as a result of tumour FGF23 overproduction.

The majority of tumours responsible for this condition are phosphaturic mesenchymal tumours (PMTs) of the mixed connective tissue variant ${ }^{2}$. These tumours are often small, slow growing, occur in diverse locations and are largely benign, however, can metastasise. Other tumours associated include osteosarcomas and advanced metastatic cancers of the colon and prostate.

\section{Imaging studies}

Following the identification of a raised FGF23 level functional imaging was undertaken to identify the possible source of a FGF23-producing tumour. PMTs are highly vascular tumours that express somatisation receptors (subtype 2A), which can be identified via an octreotide scan (figure 1) ${ }^{3}$.

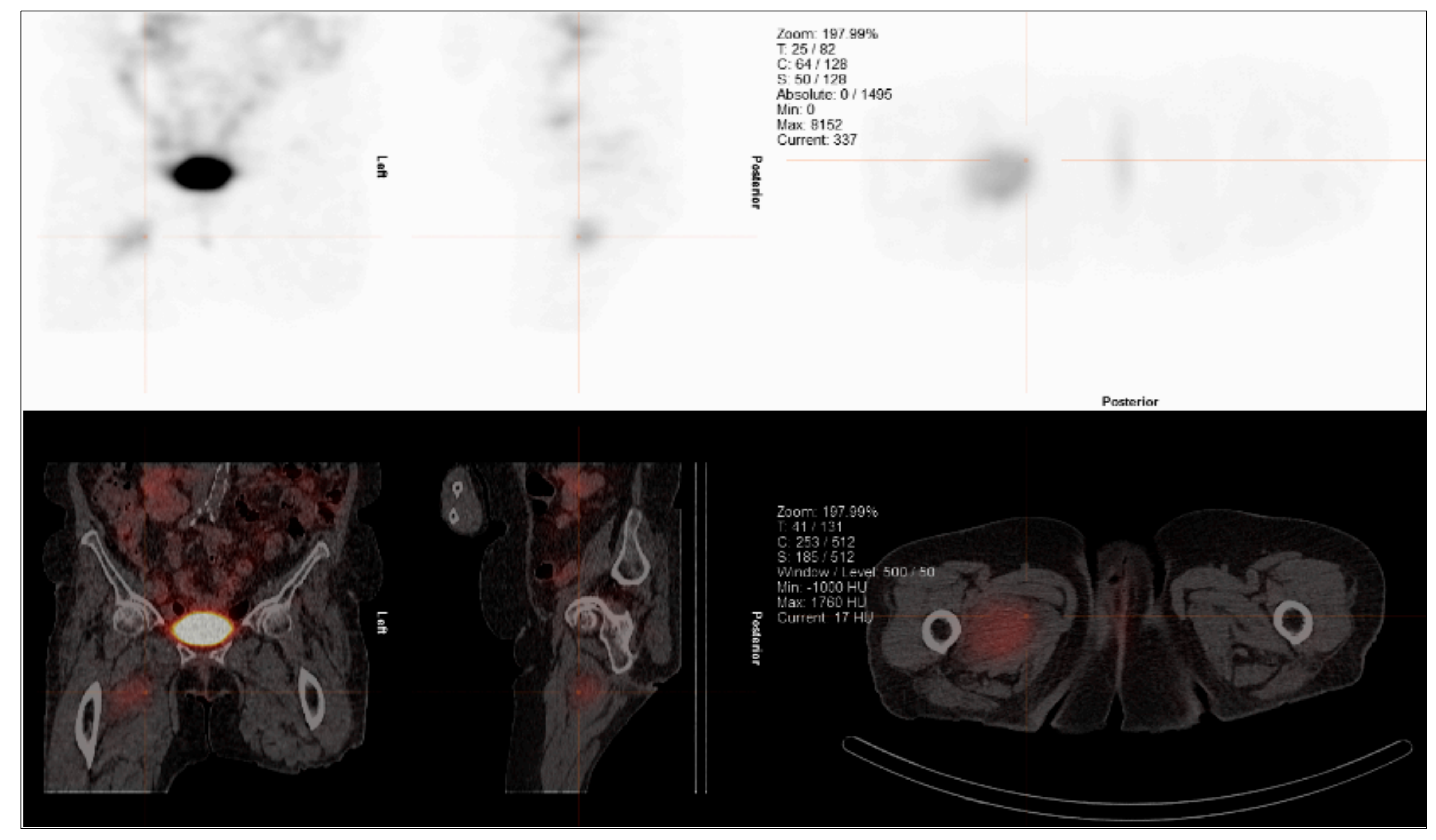

Figure 1. Octreotide Scan/SPECT CT

Within the medial aspect of the upper right thigh there is a heterogeneous soft tissue mass lesion measuring $4.2 \times 6.6 \mathrm{~cm}$ displacing adjacent musculature with corresponding mild to moderate Octreotide uptake.

A MRI of the right thigh was then undertaken. This demonstrated a well-defined infiltrative enhancing lesion in the right proximal thigh within the adductor musculature (figure 2). This patient has been referred for consideration of surgical resection.

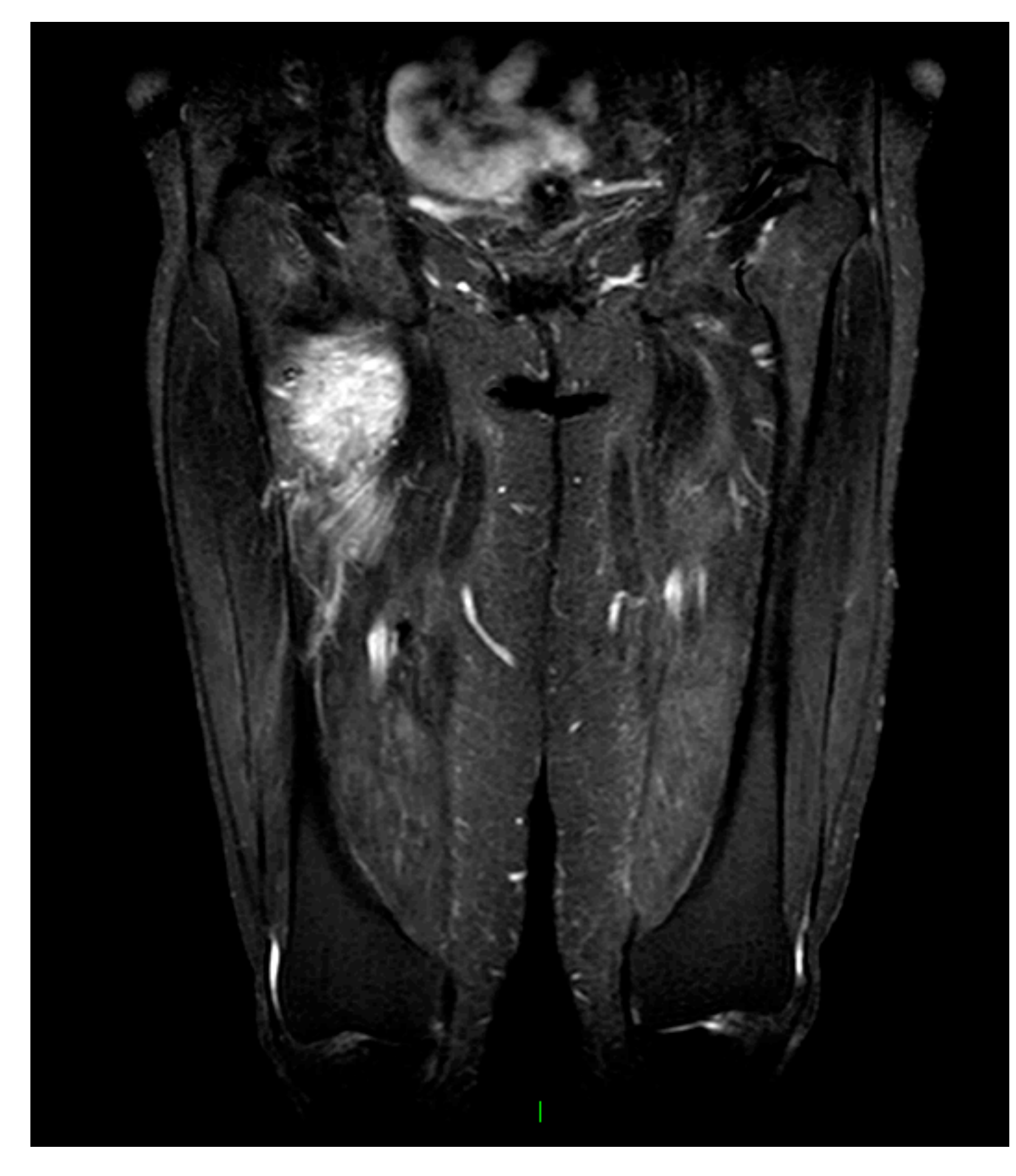

Figure 2. MRI of the right thigh. There is a $7.2 \times 3.8 \times 6 \mathrm{~cm}$ enhancing mass in the medial aspect of the right proximal thigh lying within the adductor musculature. This mass arises within the adductor brevis muscle, displacing the adjacent adductor magnus and adductor longus muscles.

\section{Conclusion}

Chronic hypophosphataemia impairs bone mineralisation and can result in significant proximal myopathy, however, patients are often asymptomatic and phosphate depletion is identified incidentally. Consequently, FGF23 is a useful biomarker in the diagnosis of tumour-induced osteomalacia, which if resected can be curative.

\section{References}

Chong WH, et al. Tumor-induced osteomalacia. Endocr Relat Cancer. 2011;18(3):R53-77

Minisola S, et al. Tumour-induced osteomalacia. Nat Rev Dis Primers. 2017; 13(3): 17044

Chong WH, et al. Tumor Localization and Biochemical Response to Cure in Tumor-Induced Osteomalacia. J Bone Minder Res. 2013; 28(6): 1386-1398 